\title{
Crystalline Morphology Developing from Cholesteric Mesophase in Cyanoethyl Chitosan Solutions
}

\author{
Yanming Dong, ${ }^{\dagger}$ Qing YuAn, Yusong Wu, Jianwei WANG, and Mian WANG \\ Department of Materials Science and State Key Laboratory for Physical Chemistry of Solid Surfaces, \\ Xiamen Universtity, Xiamen, 361005, People's Republic of China
}

(Received June 22, 1999)

\begin{abstract}
Two completely different crystals can grow from cyanoethyl chitosan/formic acid solutions in which the anisotropic liquid crystalline (LC) and isotropic phases coexisted. One was normal spherulites developing from isotropic region, and the other was cholesteric-like crystallites developing from mesophase region. The latter had similar optical characters as fingerprint texture. This implies that the crystal structure in solution cast film from cholesteric LC phase remained the main structure characters of cholesteric LC texture. A model is presented to explain the crystallization process from these liquid crystalline polymer solutions.
\end{abstract}

KEY WORDS Chitosan / Cyanoethyl Chitosan / Cholesteric Liquid Crystal / Crystalline Morphology /

Chitin and its derivatives are semi-rigid chain polymers and form lyotropic liquid crystals in appropriate solvents. Most liquid crystals formed from chitin and chitin derivatives are cholesteric. ${ }^{1-3}$ A typical texture of the cholesteric phase in chitin derivative solutions was fingerprint texture. This texture can be frozen-in by fast drying. Chitin derivative films cast from cholesteric liquid crystalline solutions exhibit regular crystalline morphology. ${ }^{4}$ But the relationship between fingerprint texture and crystalline morphology, and developing process from cholesteric order of these solutions to crystalline order of the films during casting have not been fully understood.

Cyanoethyl chitosan is a novel liquid crystalline natural polymer, and crystallizes very readily. So it benefits the investigation on both the interesting crystalline morphology of films cast from cyanoethyl chitosan/formic acid solution with polarizing optical microscope(POM), scanning electron microscope (SEM) and small-angle light scattering (SALS), and the growing process of these crystals from cholesteric mesophase by controlling the drying velocity of cast films.

\section{EXPERIMENTAL}

$O$-Cyanoethyl chitosan (CNCS) was prepared by the reaction of chitosan (deacetylation $70 \%$, viscosity 1000 $\mathrm{mPa} \mathrm{s}$ in both $1 \%$ chitosan and acetic acid solution) and acrylonitrile at room temperature using alkali preswelled method. ${ }^{5}$ The chemical structure is as follows :

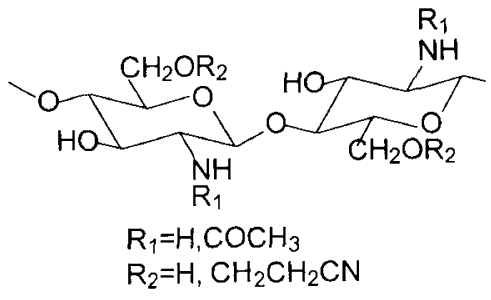

Scheme 1. The schematic illustration of chemical structure of CNCS.

\footnotetext{
${ }^{\dagger}$ To whom all correspondence should be addressed.
}

The degree of substitution, 0.77 , was measured with a HERAEUS CHN-O-RAPID elemental analyzer and Bruker DRX-400 ${ }^{1} \mathrm{H}$ NMR spectrometer at $400 \mathrm{MHz}$.

$20-22 \%(\mathrm{w} / \mathrm{w} \%)$ CNCS solution in formic acid (FA) (above $9 \%$, critical concentration forming liquid crystalline phase) was prepared and sealed in a test tube. The solution aged for 1 day at $50^{\circ} \mathrm{C}$ and subsequently 7 days at room temperature before use. The solution was then sandwiched between two glass slides. After 1 month (except especially mentioned), the sample cells were observed by OLYMPUS POM. HITACHI S520 SEM and LS-1 SALS were also used to observe the structure.

One of the slides was removed to expose the film surface for SEM measurement without etching. The contrast between crystal and amorphous in the film was enough because of the solvent evaporation during crystallization.

\section{RESULTS AND DISCUSSION}

\section{Normal Spherulites}

Like most polymers, the derivative of natural polymer CNCS formed spherulites from thick isotropic solution. Even in the solution with concentration slightly larger than the critical concentration forming liquid crystalline phase, the spherulites still appeared in the isotropic region of biphase co-exist structure.

From Figure 1, it can be seen that the spherulites consist of crystallites almost growing radially.

\section{Cholesteric-Like Crystallites}

A typical fingerprint texture can be observed in CNCS/FA solutions with concentration higher than $9 \%$ (Figure 2). This implies a cholesteric mesophase present in these solutions.

When crystallization occurred in mesophase, some interesting morphology emerged. Figure 3 a showed a typical cholesteric-like morphology in $22 \%$ CNCS/FA solution. SEM photograph (Figure $3 b$ ) revealed details of this crystal. The lamella packed in ring form. A helical disclination was clearly observed.

Figure 4 shows crystallites with cholesteric-like mor- 


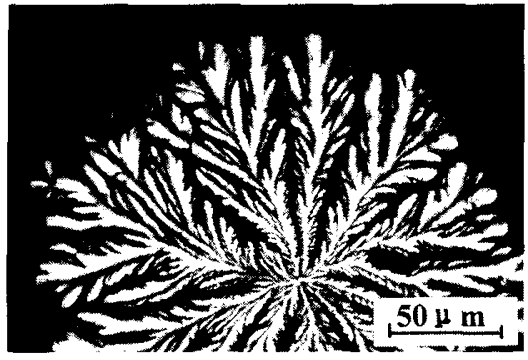

Figure 1. Polarizing micrograph of a spherulite in film cast from $20 \% \mathrm{CNCS} / \mathrm{FA}$ solution

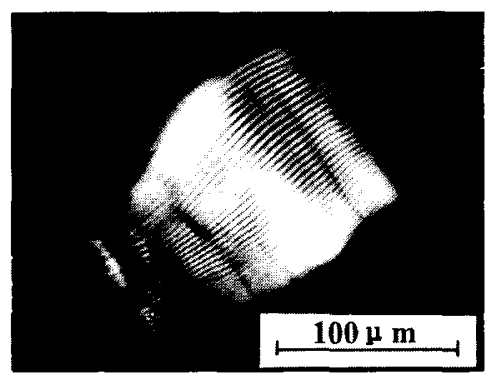

Figure 2. Polarizing micrograph of typical fingerprint texture in $20 \%$ CNCS/FA solution.
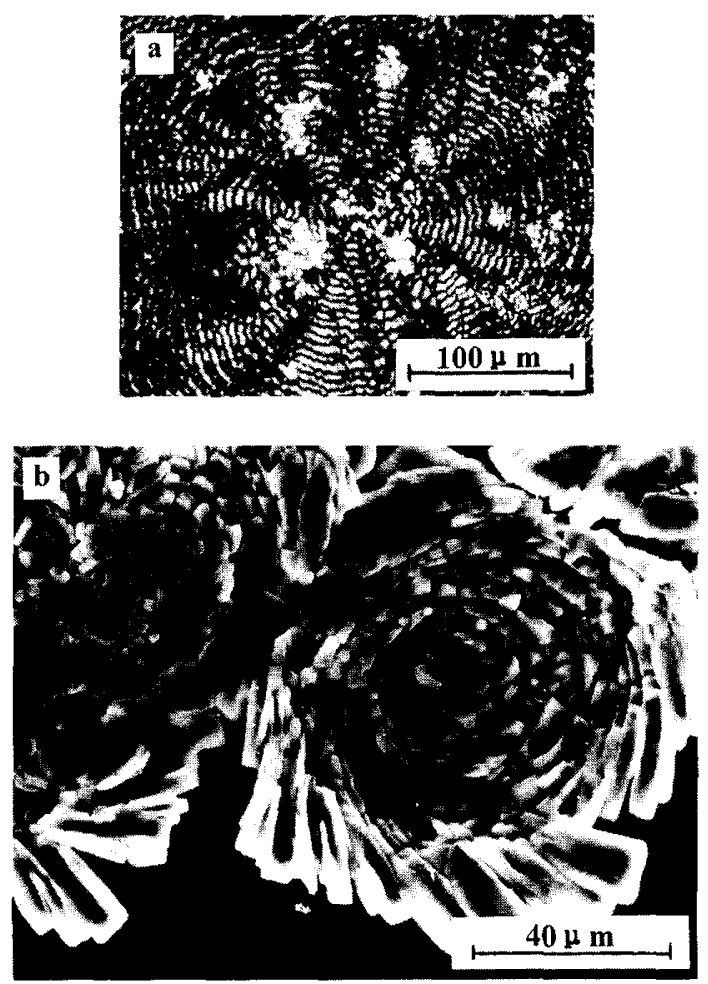

Figure 3. The cholesteric-like crystallites growing from $22 \%$ CNCS/FA solution. (a) polarizing micrograph; (b) scanning electron micrograph.

phology (region B) growing from the original texture of cholesteric liquid crystalline phase with equidistant retardation lines, the fingerprint texture (region A). The crystallites had much more contrast than liquid crystalline texture, and can be distinguished in Figure 3a. The space of retardation lines did not vary during crystallization. Molecular chains in cholesteric liquid crystalline

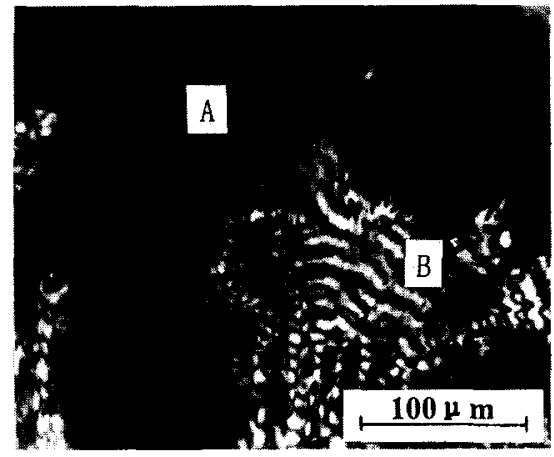

Figure 4. The fingerprint textures and cholesteric-like coexisted in $20 \% \mathrm{CNCS} / \mathrm{FA}$ solution cast film, showing the growing process of the latter and the relationship between these two structures.
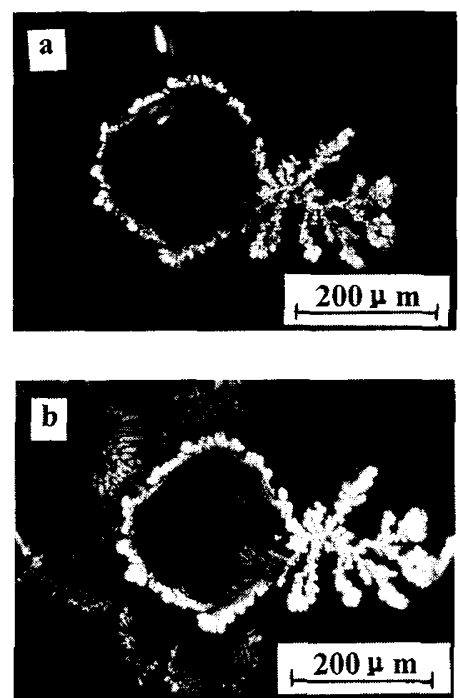

Figure 5. The growth of cholesteric-like crystallites. (a) A spherulite existing in isotropic region of $20 \%$ CNCS/FA solution after preliminarily drying at $50^{\circ} \mathrm{C}$ for $3 \mathrm{~d}$; (b) The cholesteric-like crystallites developed from the microstructure of (a) after further drying for 7 days at room temperature.

phase may crystallize in situ by adjusting the directors among adjacent layers slightly.

Other direct evidence that cholesterc-like crystallites grow from mesophase is illustrated in Figure 5. The cholesteric-like crystallites were observed to develop aside a normal spherulite during further drying of cast solution film, because the solution in this region turned into a mesophase as the concentration became higher.

SALS measurements of cholesteric-like crystallites showed a very similar pattern as fingerprint textures ${ }^{6}$ (Figure 6). SALS determination of pitch in fingerprint texture $^{7}$ also can be used to measure the twisted period of cholesteric-like crystallites. The twisted period obtained from Figure $6 \mathrm{~b}$ was $15 \mu \mathrm{m}$, which accords with the average result $(\sim 15 \mu \mathrm{m})$ of POM (Figure $6 \mathrm{a}$ ).

Some cholesteric-like crystallites exhibited a lamella structure in which the layers were quite straight (Figure 7a). They crystallized from the straight fingerprint texture (See Figure 1) often existing in chitin-based polymer due to high rigidity of the molecular chain. ${ }^{8}$ SEM photograph (Figure 7b) illustrates the details of these structures. Every layer is composed of crystallites in 2 $\mu \mathrm{m}$ or so. This microstructure can be regarded as a spe- 

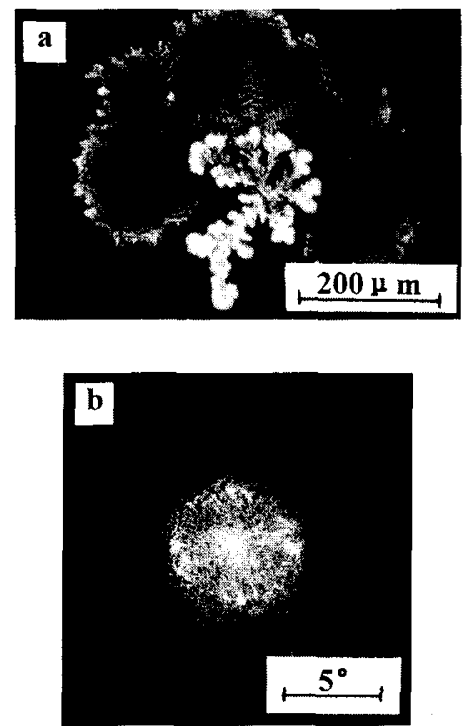

Figure 6. Polarizing micrograph of typical cholesteric-like crystallites of CNCS and its correspoding SALS pattern. (a) polarizing micrograph ; (b) SALS pattern.
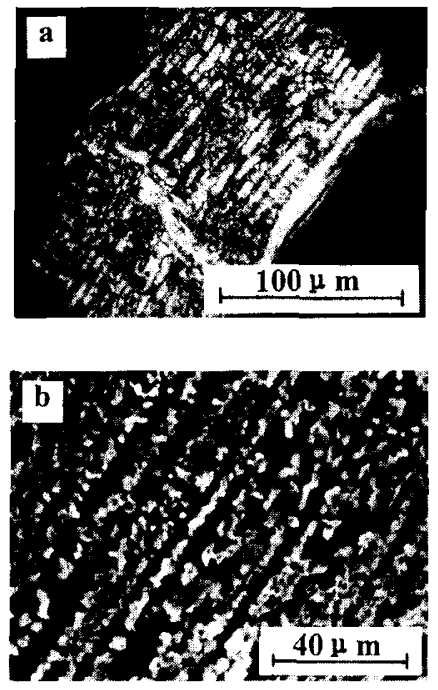

Figure 7. A lamella structure with cholesteric-like crystallites growing from $22 \%$ CNCS/FA solution. (a) polarizing micrograph ; (b) scanning electron micrograph.

cial case of cholesteric-like crystallites.

\section{Model of Cholesteric-like Crystallites}

The optical properties of birefringence in three microstructures, i.e., fingerprint texture, spherulites, cholesteric-like crystallites, can be detected by POM using a $\lambda$ gypsum plate. The results of fingerprint texture (Figure 8) showed that the direction of molecular length is the slow axis. Molecular direction (director) in the two latter microtructures can thus be determined. In spherulites, the directors were parallel to the radius, i.e, the growing direction. In cholesteric-like crystallites the directors were perpendicular to the direction of retardation lines, i.e., vertical to the growing direction. This means that the growing process and the aggregation mechanism of crystallites completely differ in isotropic and anisotropic phases. A scheme illustration for possible crystallization process of CNCS/FA solution is pre-

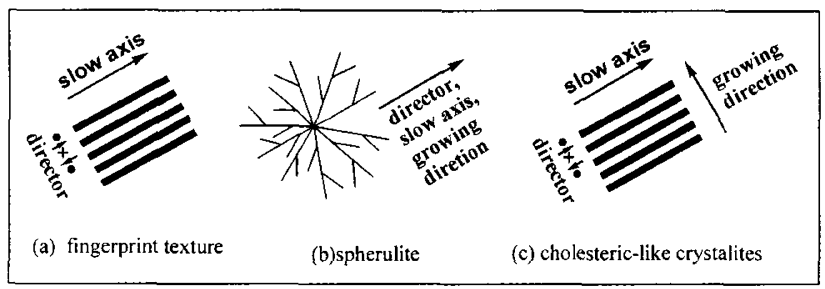

Figure 8. The optical properties of birefringence and directors in fingerprint texture, spherulites and cholesteric-like crystallites measure by POM.

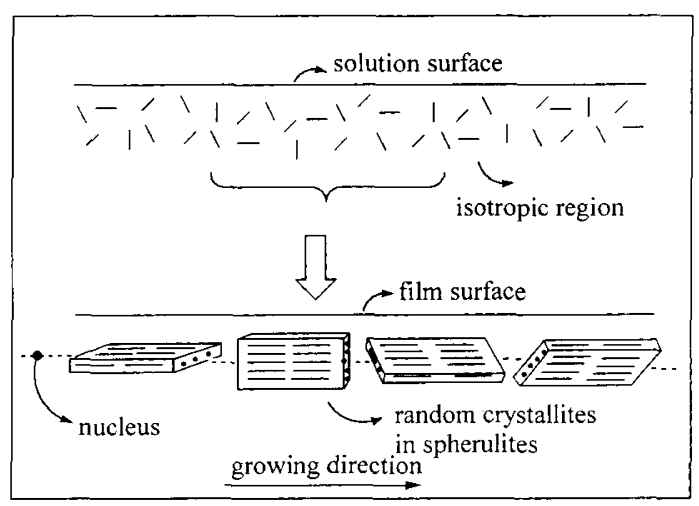

(a) the formation of spherulites

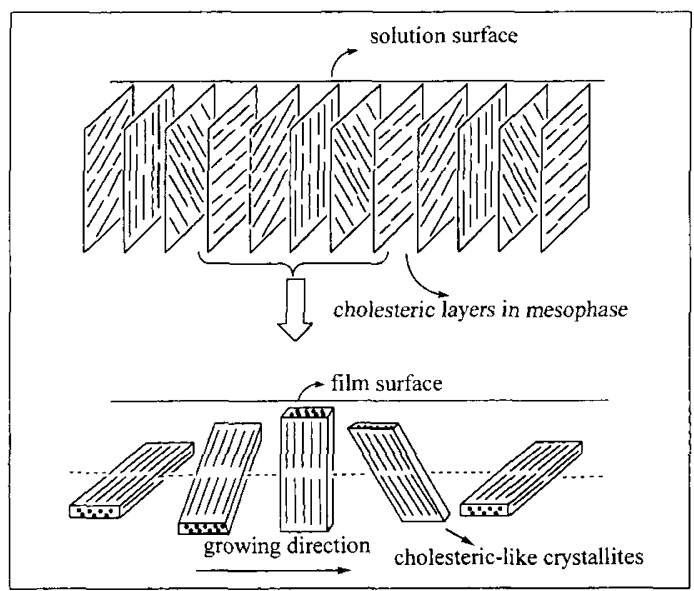

(b) the formation of cholesteric-like crystallites

Figure 9. The scheme for possible crystallization process in CNCS/FA cholesteric liquid crystalline solution.

sented in Figure 9.

Acknowledgment. This research was supported by National Natural Science Foundation of China and Natural Science Foundation of Fujian, China.

\section{REFERENCES}

1. K. Ogura, T. Kanamoto, T. Sanan, K. Tanaka, and Y. Iwakura. Proc. 2nd Int. Conf. Chitin/chitosan, Tottori, Japan, 1982, p 39.

2. D. K. Rout, S. P. Pulapura, and R. A. Gross. Macromolecules, 26,5999 (1993).

3. Y. Dong and J. Zhang. Chem. J. Chin. Univ., 17, 973 (1996),

4. Y. Dong and Z. Li. Guangzhou Chem., 4, 24 (1997).

5. S. Tokura, N. Nishi, S. Nishimura, and Y. Ikeuchi. Polym. J., 


\section{Crystalline Morphology Developing from Mesophase}

15, 553 (1983).

6. Y. Onogi, J. L. White, and J. F. Fellers. J. Polym. Sci., Phy. $E d ., 18,663$ (1980).
7. Y. Dong and Z. Li, Polym. J., 30, 272 (1998).

8. M. Terbojevich, A. Cosani, G. Conio, E. Marsano, and E. Bianchi, Carbohydr. Res., 209, 251 (1991). 